\section{Cohen's kappa coefficient of observer agreement: A BASIC program for minicomputers}

\author{
D. R. WIXON \\ Clark University, Worcester, Massachusetts 01610
}

Reliability of measurement is a basic problem in psychology. Despite the fact that better measures exist, many investigators still use percent agreement as an index of reliability of nominal classification system (e.g., Sagotsky, Patterson, \& Lepper, 1978). Cohen (1960) has suggested an alternative (kappa) that overcomes the problems inherent in the percent agreement and $\chi^{2}$ approaches to nominal data. Although the computation of a single kappa is relatively straightforward, its repeated computation, particularly when several categories are involved, may be tedious. The program presented here relieves that tedium and provides the user with virtually all the computations relevant to kappa. In addition, the present program differs from previously published programs (Berk \& Campbell, 1976) in that an interactive input is provided and revised formulas (Fleiss, Cohen, \& Everitt, 1969) are used to calculate the variance of kappa and provide an error term for the $z$ test.

Input. Initial input is the number of categories in the classification system; up to 10 categories $^{1}$ are allowed in the present version. Next, the program requests the observed frequency of judgments in each cell of the twodimensional array formed by the classification system used by the two judges. The options of printing the raw cell frequencies (with marginal totals) or correcting any cell frequency are presented repeatedly until the option of performing the final computations is chosen.

Output. The output, including the optional printing of observed frequencies, is presented in Table 1. The output includes the observed frequencies of agreement between judges, the frequency of agreement expected by chance, the kappa, the standard error of the kappa, the $\mathrm{z}$ value for a significance test of the kappa, and the maximum kappa that could occur given the marginal totals. (Except where explicitly noted, formulas used are taken directly from Cohen, 1960.)

Program Language and Requirements. The program uses only $3.37 \mathrm{~K}$ bytes of core as implemented on a WANG 2200-A. The program is written in a form of BASIC that does not require the specification of LET and allows PRINTUSING and INPUT statements. Extensive use of continuation statements, using the standard
Table 1

Sample Output

OBSERVED FREQUENCIES

JUDGE B JUDGE A

$\begin{array}{rrrrr} & 1 . & 2 . & 3 . & \\ 1 . & 88 . & 14 . & 18 . & 120 . \\ 2 . & 10 . & 40 . & 10 . & 60 . \\ 3 . & 2 . & 6 . & 12 . & 20 . \\ & 100 . & 60 . & 40 . & \end{array}$

$\begin{array}{rcc}\text { AGREEMENT } & & \\ \text { CATEGORY } & \text { OBSERVED } & \text { EXPECTED } \\ 1 . & 88 . & 60.00 \\ 2 . & 40 . & 18.00 \\ 3 . & 12 . & 4.00\end{array}$

KAPPA $=.4915254237288$

STANDARD ERROR $=5.10018155 \mathrm{E}-02$

Z-VALUE $=9.456242435453$

MAXIMUM $\mathrm{K}=.8305084745763$

Note-These are sample data taken from Cohen $(1960$, p. 45).

colon $(:)$ delimiter, results in a program that is only 60 statements long, including REM statements. The current version uses both a CRT and a printer as output devices, but that could be changed. Following the initial clearing of variables (requiring less than $2 \mathrm{sec}$ ), execution time is primarily related to input/output time.

Availability. Program listings will be provided at no cost on request to the author, D. R. Wixon, Department of Psychology, Clark University, Worcester, Massachusetts 01610.

\section{REFERENCES}

Berk, R. A., \& CAMpbell, K. L. A FORTRAN program for Cohen's kappa coefficient of observer agreement. Behavior Research Methods \& Instrumentation, 1976, 8, 396.

Cohen, J. A coefficient of agreement for nominal scales. Educational and Psychological Measurement, 1960, 20, 37-46.

Fleiss, J. L., Cohen, J., \& Everitt, B. S. Large sample standard errors of kappa and weighted kappa. Psychological Bulletin, $1969,72,323-327$.

Sagotsky, G., Pattenson, C. J., \& Lepper, M. P. Training children's self-control: A field experiment in self-monitoring and goal-setting in the classroom. Journal of Experimental Child Psychology, 1978, 25, 242-253.

\section{NOTE}

1. A second program with slightly revised input requirements and output formats will allow for up to 50 categories. A listing will be provided at no cost on request to the author.

(Accepted for publication October 20, 1979.) 